\title{
Cosmological models and the brightness profile of distant galaxies
}

\author{
I. Olivares-Salaverri ${ }^{1}$ and Marcelo B. Ribeiro ${ }^{2}$ \\ ${ }^{1}$ Observatório do Valongo, Universidade Federal do Rio de Janeiro, Brazil; iker@astro.ufrj.br \\ ${ }^{2}$ Instituto de Física, Universidade Federal do Rio de Janeiro, Brazil; mbr@if.ufrj.br
}

This work aims to determine the feasibility of an assumed cosmological model by means of a detailed analysis of the brightness profiles of distant galaxies. Starting from the theory of Ellis \& Perry (1979) connecting the angular diameter distance obtained from a relativistic cosmological model and the detailed photometry of galaxies, we assume the presently most accepted cosmology with $\Lambda \neq 0$ and seek to predict the brightness profile of a galaxy in a given redshift $z$. To do so, we have to make assumptions concerning the galactic brightness structure and evolution, assuming a scenario where the specific emitted surface brightness $B_{e, \nu_{e}}$ can be characterized as, $B_{e, \nu_{e}}(r, z)=B_{0}(z) J\left(\nu_{e}, z\right) f[r(z) / a(z)]$. Here $r$ is the intrinsic galactic radius, $\nu_{e}$ the emitted frequency, $B_{0}(z)$ the central surface brightness, $J\left(\nu_{e}, z\right)$ the spectral energy distribution (SED), $f[r(z) / a(z)]$ characterizes the shape of the surface profile distribution and $a(z)$ is the scaling radius. The dependence on $z$ is due to the galactic evolution. As spacetime curvature affects the received surface brightness, the reciprocity theorem (Ellis 1971) allows us to predict the theoretical received surface brightness. So, we are able to compare the theoretical surface brightness with its equivalent observational data already available for high redshift galaxies in order to test the consistency of the assumed cosmological model. The function $f[r(z) / a(z)]$ is represented in the literature by various different shapes, like the Hubble, Hubble-Oemler and Abell-Mihalas single parameter profiles, characterizing the galactic surface brightness quite well when the disk or bulge dependence is dominant. Sérsic and core-Sérsic profiles use two or more parameters and reproduce the galactic profile almost exactly (Trujillo et al. 2004). If we consider all wavelengths, the theory tells us that the total intensity is equal to the surface brightness, so the chosen bandwidth should include most of the SED. In order to analyze only the effect of the cosmological model in the surface brightness and minimize evolutionary effects, we assume that there exists a homogeneous class of objects, whose properties are similar in all redshifts, allowing us to carry out comparisons at different values of $z$. Studying the parameters that affect the galactic evolution, as well as in others geometrical tests, we will be able to infer some possible galaxy evolution which could reproduce a theoretical surface brightness profile, in order to compare with the observational data and reach conclusions about the observational feasibility of the underlying cosmological model.

Keywords. galaxies: structure, galaxies: evolution, cosmology: observations.

\section{References}

Abell, G. O \& Mihalas, D. M 1966, AJ 71, 7

Binney, J. \& Merrifield, M. 1999, Galactic Astronomy

Ellis G. F. R. 1971, in: Sachs, R. K. (ed.) Proc. Int. School of Physics "Enrico Fermi": pp. 104-182. Academic Press, New York (1971). Reprinted: Gen. Relativ. Grav. (2009) 41,581

Ellis, G. F. R. \& Perry, J. J. 1979, Mon. Not. R. astr. Soc. (1979) 187, 357

Trujillo, I., Peter Erwin, Asensio Ramos, A., \& Graham, A. W. 2004, AJ 127, 1917 